\title{
Lost in translational filters between peers
}

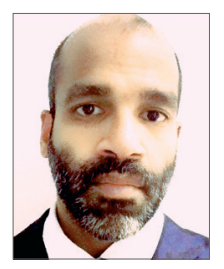

Kumaran Kolandaivelu ${ }^{1,2 *}$, MD, PhD; Abraham R. Tzafriri ${ }^{3}, \mathrm{PhD}$

1. Institute for Medical Engineering and Science, Massachusetts Institute of Technology, Cambridge, MA, USA;

2. Cardiovascular Division, Brigham and Women's Hospital, Harvard Medical School, Boston, MA, USA; 3. CBSET, Lexington, MA, USA

In a recent issue of EuroIntervention, two articles ${ }^{1,2}$ and an editorial $^{3}$ detailed the translational journey taken by Qvanteq's ultra-hydrophilic stent platform. Bench-top studies and animal experiments in two species of swine as well as rabbits suggested that a reduction in restenosis and stent thrombosis might be anticipated based on preclinical indices. In contrast, the first-in-man (FIM) study did not corroborate these results. Ultra-hydrophilic devices had a rate of neointimal formation similar to that found in contemporary bare metal stents (BMS). Rightfully, these reports question the relevance of preclinical models when ultimate risk/ benefit plays out in humans with disease.

As translational researchers and authors of the preclinical animal work, we praise EuroIntervention and the Editors for curating this topic. Such timed alignment in bridging articles is rare, let alone in a single, high-impact forum. Advances in transgenic animal models ${ }^{4}$ and emerging CRISPR technologies ${ }^{5}$, coupled with the ability to correlate disease, treatment, and response in vivo with exquisite measurement tools, demand that we rethink together how best to configure the translational "filters" between animal and human. If suboptimal technologies pass, patients are exposed to undue risks. If promising technologies are sifted prematurely, putative benefits and investments are lost. Given the stakes, we feel it is crucial to comment on how divergent preclinical and clinical conclusions could have been reached. In so doing, we hope to extend hands between preclinical and clinical colleagues and advance much needed interdisciplinary, inter-sector, and international debate.
The FIM study was master-class assessment of ultra-hydrophilic stent performance in situ. Using high-resolution OCT imaging and analysis, Suwannasom et al created stellar mappings between strut embedding (stimulus) and neointimal thickness (response). In doing so, they propel our joint ability to correlate preclinical and clinical domains. Perhaps most importantly, they unmasked an unknown safety concern and made the judicious call to minimise patient risk by stopping the study early ( 31 of 35 planned patients) after observing unanticipated stent dislodgements. We also agree fully with author and editorial sentiments regarding the inaccuracies of healthy animal models. Device implantation sites in healthy animal arteries are less accurate and less variable representations of the anatomies, compositions and injury patterns observed in disease. Given available large animal disease models, is there value in simpler systems?

As preclinical and clinical researchers, we are all challenged with the reality that all models, by definition, are wrong. This holds for rabbits, Yorkshire swine, Yucatan mini-pigs ${ }^{6}$, CRISPR ApoE/ LDLR double knockouts, or even the entirety of clinical experiences prior to managing a new case. The most relevant questions of any model can only be whether it is useful and the level of uncertainty one should pass forward. Using healthy animals, we were able to conduct a substantial investigation in 26 swine and six rabbits, into which 95 stents were placed (ultra-hydrophilic stents with matched BMS and drug-eluting stents [DES]). These numbers allowed us to consider three stent platforms and four time points. Rather than detail restenosis spatially, we quantified it dynamically and observed a maximal ultra-hydrophilic benefit in neointimal

*Corresponding author: MIT-IMES Clinical Research Center, E25-201, 77 Massachusetts Avenue, Cambridge, MA 02139, USA.E-mail: kkolandaivelu@bwh.harvard.edu 
reduction at the 30-day assessment that seemed to close by day 90. If true, the six-month non-superiority FIM finding need not be contradictory.

It was in this 30-day window that we also observed improved endothelialisation in ultra-hydrophilic platforms over DES, with scattered, non-significant delays in BMS endothelialisation (classified as outliers). We were intrigued that high thrombotic indices were only observed in cases of suboptimal endothelialisation and that all ultra-hydrophilic devices endothelialised rapidly and irrespective of animal model used (and as anticipated from cell culture ${ }^{7}$ ). Here too, that the only definite FIM stent thrombosis case occurred in a non-study BMS, which was deployed in the setting of stent dislodgement, seems in alignment with preclinical observations given that far more FIM ultra-hydrophilic devices were implanted, none of which thrombosed. Is the non-study case random noise and best forgotten? Or is it an outlying event that may hold mechanistic insight or which could take years to manifest in the clinic?

\section{The knowledge and uncertainty lost in translation}

Without specific comment on the risks and benefits of Qvanteq's ultra-hydrophilic approach, we submit the peer review journey of our manuscript and our reflections on how information may have been lost in translation. Prior to being shepherded by the Editors of EuroIntervention, our report underwent a two-year process of peer reviews and rejections. While refining and clarifying the statistically significant findings, details were minimised or lost altogether through progressive cuts. One such cut stated:

"Intriguingly, the 'frequency' of the rabbit model outlier $(1 / 12=0.083)$ was similar to the frequency of outlying suboptimal healing/platelet responses observed in porcine studies $(4 / 51=0.078)$. Assuming an event rate of 0.08 , the Poisson probability of observing no outlying responses in 25 UHS samples (19 porcine +6 rabbit) was 0.135 , suggesting a trend to outlier reduction compared to next generation stent platforms."

Statements, where p-values failed to achieve traditional cutoffs, were forced out as we struggled to pass forward knowledge and uncertainties across the translational divide. Where else (from finance and insurance to gambling and art) would a certainty of $51 \%$ not be perceived as useful knowledge, let alone $86.5 \%$ ?

Perhaps the unique aspect of our preclinical study, other than providing the first in vivo demonstration of a viable technology, was the use of modest animal numbers to make exploratory, statistical comments on rare thrombotic trends. Recent history has taught that, on a backdrop of atherosclerotic "noise" (human or animal), detecting such events can take thousands of subjects. There is a significant cost burden associated with conducting major studies in healthy animals. This cost is amplified significantly in diseased large animal models that are $>10 \mathrm{x}$ more expensive. More explicitly, a $\$ 100,000$ study would cost over $\$ 1 \mathrm{M}$; a $\$ 200,000$ study would cost over $\$ 2 \mathrm{M}$, etc.

Translational endeavours cut across disciplinary sectors and are carried out by researchers with different backgrounds, interests, motivations, and budgets. As we try to speak to one another through established peer-reviewed mechanisms, we are faced with one of two decisions: 1) publish in highly specialised expert journals and miss target audiences at a different translational step, or 2) avoid use of nuanced jargon and complex results at a time when nuance and complexity are still important. Even more problematic, if communication pathways are gated by p-values, unimaginable amounts of knowledge, insight, and value have been and will continue to be lost. Translation is not simple - it is complex. As we continue to refine preclinical approaches and develop strategies governing judicious use of more accurate but more expensive disease models, it is equally important to continue exploring mechanisms to promote the effective transfer of gained knowledge and uncertainties.

\section{Conflict of interest statement}

The authors have no conflicts of interest to declare.

\section{References}

1. Kolandaivelu K, Bailey L, Buzzi S, Zucker A, Milleret V, Ziogas A, Ehrbar M, Khattab AA, Stanley JRL, Wong GK, Zani B, Markham PM, Tzafriri AR, Bhatt DL, Edelman ER. Ultrahydrophilic stent platforms promote early vascular healing and minimise late tissue response: a potential alternative to secondgeneration drug-eluting stents. EuroIntervention. 2017;12: 2148-56.

2. Suwannasom P, Sotomi Y, Corti R, Kurz DJ, Roffi M, von Birgelen C, Buzzi S, Zucker A, Dijkstra J, Wykrzykowska JJ, de Winter RJ, Windecker S, Onuma Y, Serruys PW, Daemen J, Räber L. First-in-man six-month results of a surface-modified coronary stent system in native coronary stenosis. EuroIntervention. 2017; 12:2118-27.

3. Khamis R. The translational journey of a new ultra-hydrophilic stent platform: what can we learn? EuroIntervention. 2017;12:2044-5.

4. Li Y, Fuchimoto D, Sudo M, Haruta H, Lin QF, Takayama T, Morita S, Nochi T, Suzuki S, Sembon S, Nakai M, Kojima M, Iwamoto $\mathrm{M}$, Hashimoto $\mathrm{M}$, Yoda $\mathrm{S}$, Kunimoto $\mathrm{S}$, Hiro $\mathrm{T}$, Matsumoto T, Mitsumata M, Sugitani M, Saito S, Hirayama A, Onishi A. Development of Human-Like Advanced Coronary Plaques in Low-Density Lipoprotein Receptor Knockout Pigs and Justification for Statin Treatment Before Formation of Atherosclerotic Plaques. J Am Heart Assoc. 2016;5:e002779.

5. Huang L, Hua Z, Xiao H, Cheng Y, Xu K, Gao Q, Xia Y, Liu Y, Zhang X, Zheng X, Mu Y, Li K. CRISPR/Cas9-mediated ApoE-/- and LDLR-/- double gene knockout in pigs elevates serum LDL-C and TC levels. Oncotarget. 2017;8:37751-60.

6. Agarwala A, Billheimer J, Rader DJ. Mighty minipig in fight against cardiovascular disease. Sci Transl Med. 2013;5:166fs1.

7. Milleret V, Ziogas A, Buzzi S, Heuberger R, Zucker A, Ehrbar M. Effect of oxide layer modification of $\mathrm{CoCr}$ stent alloys on blood activation and endothelial behavior. J Biomed Mater Res B Appl Biomater. 2015;103:629-40. 